\title{
¿Pueden los centros de Formación Profesional tener un papel en el sistema de innovación? El caso de Navarra /
}

\section{Could Vocational and Training institutes Play a Role in the Innovation System? The Case of the Navarre Region}

\author{
Beatriz Otero Gutiérrez \\ Departamento de Sociología y Trabajo Social. \\ Universidad del País Vasco (UPV/EHU). España / Spain \\ beatriz.otero@ehu.eus \\ Cristina Lavía \\ Departamento de Sociología y Trabajo Social. \\ Universidad del País Vasco (UPV/EHU). España / Spain \\ cristina.lavia@ehu.eus
}

Recibido / Received: 21/10/2015

Aceptado / Accepted: 26/05/2016

\section{RESUMEN}

El papel de los centros de Formación Profesional (FP) en el desarrollo económico y la innovación no ha recibido una atención suficiente en los estudios recientes sobre los sistemas regionales de innovación. En este artículo, basado en una encuesta a 219 pymes industriales, se analiza la situación de Navarra, una comunidad autónoma española que presenta importantes niveles de desarrollo de la educación profesional y de relación de la misma con las empresas. Se analiza el papel del personal de FP en la empresa y, específicamente, su participación en los procesos de innovación. Posteriormente, se pretende averiguar si las relaciones que las empresas establecen con los centros de FP pueden incidir en las actividades de innovación. En este sentido, se muestra que la realización de actividades de formación continua ejerce un efecto multiplicador sobre la probabilidad de participación de los trabajadores en la innovación.

Palabras clave: Formación profesional, pymes, innovación, región, participación de los trabajadores/as.

\author{
Mikel Olazaran Rodríguez. \\ Departamento de Sociología y Trabajo Social. \\ Universidad del País Vasco (UPV/EHU). España / Spain \\ mikel.olazaran@ehu.eus \\ Eneka Albizu \\ Departamento de Economia Financiera II. \\ Universidad del País Vasco (UPV/EHU). España / Spain \\ eneka.albizu@ehu.eus
}

\section{ABSTRACT}

Recent research from the innovation system perspective has dedicated scant attention to the role of VET institutes and workers in innovation processes. This paper, based on a survey to 219 industrial SMEs, looks at the case of Navarre, an autonomous region from Northern Spain which has a long tradition of relationships between VET education and industry. First of all, attention is paid to the firms' perception of the VET offer and to the different types of relationships between VET institutes and firms. Then an attempt is made to measure the contribution of VET personnel to SME's innovation activities. Finally, we check whether the more developed forms of relationships between VET institutes and firms can have an effect upon the latter's innovation activities. Using a logistic regression, it is shown that contracting continuous vocational training activities with the VET institutes has a multiplying effect upon workers' participation in innovation.

Keywords: Vocational education and training, VET, SMEs, innovation, region, Spain, worker participation.

\footnotetext{
*Autor para correspondencia / Correspnding author: Beatriz Otero Gutiérrez. Departamento de Sociología y Trabajo Social. Facultad de Ciencias Sociales y de la Comunicación. Universidad del País Vasco/Euskal Herriko Unibertsitatea (UPV/EHU). Barrio Sarriena s/n. 48940 Leioa (Bizkaia). Sugerencia de cita / Suggested citation: Otero, B., Lavía, C., Olazaran, M., Albizu, E. (2016). ¿Pueden los centros de FP tener un papel en el sistema de innovación? El caso de Navarra. Revista Española de Sociología, 25 (3): 387-407.

(http://dx.doi.org/10.22325/fes/res.25.3.2016.387)
} 


\section{INTRODUCCIÓN}

La Formación Profesional (FP) es un pilar básico, no suficientemente reconocido, de la productividad, la capacidad colectiva de innovación y el progreso social en los países industrializados. En comparación con los países europeos de referencia, España presenta importantes debilidades a este respecto, con niveles inferiores de población que ha realizado estudios de secundaria postobligatoria y menores tasas de estudiantes de FP reglada en comparación con países europeos de referencia (CEDEFOP, 2010). También se cuenta con niveles tradicionalmente bajos en el ámbito de la formación continua, en términos de costes de formación respecto a los costes laborales, empresas que ofrecen formación a sus trabajadores/ as y trabajadores que participan en cursos de formación (Crespo y Sanz, 2000).

En los últimos años la proporción de empresas que realizan actividades formativas ha aumentado de forma notable, pasando del $26,5 \%$ de las empresas de 100 más trabajadores en 1993 al 74,1\% en 2010 (Ministerio de Empleo, 2013). En paralelo, la financiación pública de las actividades de formación de oferta ha crecido notablemente hasta la actual crisis y, en los últimos años, la formación de demanda, basada en el sistema de bonificaciones, ha experimentado una considerable expansión (Vargas Sampedro, 2015).

Sin embargo, a día de hoy, se sigue constatando un importante retraso respecto a la media europea en indicadores importantes como el gasto de las empresas en formación respecto al total de costes laborales ( $0,6 \%$ en España frente a $0,9 \%$ en UE-27) 0 el porcentaje de empresas innovadoras cuyo personal ha realizado actividades formativas de soporte a la innovación ( $10,4 \%$ frente a $46,4 \%$ respectivamente) (CEDEFOP, 2013). Por otro lado, el nivel de implantación de los cambios normativos recientes en la formación profesional no ha sido suficiente para superar los problemas de coordinación del sistema, certificación de cualificaciones o desarrollo de los centros integrados de FP.

En este trabajo se estudia el caso de la Comunidad Foral de Navarra, una región altamente especializada en el sector industrial, con un nivel de valor añadido bruto sobre el PIB regional del 23,5\%, 9 puntos por encima de la media española $(14,8 \%)$. Esta comunidad autónoma cuenta con una importante trayectoria de Formación Profesional y relaciones de dichos centros con las empresas que se refleja en los niveles de población ocupada con este nivel educativo $(27,3 \%$ frente a una media estatal del 19,5\%). En el sector industrial, en el que se centra este trabajo, Navarra cuenta con un porcentaje de población ocupada con perfil de FP Superior del $22,7 \%$, por encima de la media española del $15,4 \%$, siendo los niveles de FP de Grado Medio del 16,4\% y 10,5\% respectivamente.

Estudios anteriores realizados en esta región muestran que, si bien los centros de FP tienen un nivel importante de relación con el entorno regional empresarial, en lo que respecta a la provisión de capital humano, existen importantes obstáculos, tanto internos como externos a estos agentes educativos, para el desarrollo de formas "avanzadas" de relación con la empresa, como son la formación continua o los servicios técnicos, que pudieran incidir en los procesos de innovación de las empresas (Olazaran, Albizu, Lavía y Otero, 2013). En este trabajo, basado en una encuesta diseñada a tal efecto a 219 pymes industriales, se estudia la posible incidencia que la formación continua impartida por los centros de FP puede tener en los procesos de innovación de las empresas, a través de la participación de los trabajadores en los mismos.

Partiendo de una concepción amplia de la innovación, que pueda ser adecuada para pymes y para sectores de menor intensidad tecnológica, las principales preguntas a las que esta investigación pretende responder son las siguientes: ¿participan los trabajadores con perfil de FP en los procesos de innovación de las empresas?, ¿en qué tipo de innovaciones?, iexisten diferencias a este respecto entre las pymes más y menos innovadoras?, ¿inciden las relaciones con los centros de FP en una mayor participación de los trabajadores en la innovación? 0, dicho de otra manera, ¿pueden los centros de FP jugar un papel en el sistema de innovación? 
La estructur a del documento es la siguiente. En primer lugar se expone el marco teórico desde el que se realiza la investigación, donde se tratan puntos como las relaciones entre el sistema educativo-formativo de FP y el sistema de innovación, las especificidades de los procesos de innovación en pymes y sectores de menor intensidad tecnológica y el papel que las actividades de formación continua pueden tener en dichos procesos. También se revisan algunos rasgos relevantes del contexto institucional y cultural español, como son el menor prestigio de la FP respecto a la educación universitaria, los debates en torno a la oferta formativa reglada y las debilidades del sistema de formación continua.

Posteriormente, se describe la metodología empleada, basada principalmente en una encuesta telefónica a una muestra de 219 pymes industriales, detallando la composición sectorial, de tamaño y de nivel tecnológico de la misma.

Tras ello, en el apartado cuarto se cuantifica la presencia de personal de FP en las empresas y se atiende a la percepción de las mismas sobre la capacitación de los egresados de FP en el momento de acceder a la empresa y sobre la contribución de estos trabajadores a la competitividad de sus organizaciones. También se considera la valoración de las empresas sobre la oferta de FP reglada del entorno. En general, se constatan algunas diferencias significativas en la valoración del papel del personal de FP por nivel tecnológico y sector. A este respecto, se ofrece evidencia cualitativa que puede ayudar a entender las valoraciones más críticas de los sectores más relacionados con la FP.

El apartado quinto revisa los distintos tipos de relaciones que las empresas mantienen con los centros de FP. Se constata la importancia de las prácticas de Formación en Centros de Trabajo (FCT) como mecanismo de selección y contratación de personal y como forma básica de relación entre los centros formativos y las pymes. También se detecta un importante nivel de relación en actividades de formación continua. Por medio de evidencia cualitativa, se ofrecen algunos rasgos de la situación y limitaciones a este respecto.

A continuación, en el apartado sexto, se analizan las actividades de innovación realizadas en las empresas y la participación del personal de FP en las mismas. Se parte de un concepto amplio de innovación, que incluye mejoras de productos y procesos. Se constata que, a pesar de pertenecer mayoritariamente a sectores de intensidad tecnológica media o baja, las pymes encuestadas Ilevan a cabo un nivel importante de actividades de innovación, con una importante participación de los trabajadores de FP en las mismas. Se ha definido un indicador del grado de innovación (mayor o menor respecto a los competidores directos) y se ha encontrado que las empresas más innovadoras presentan un nivel de participación del personal de FP mayor que el de las empresas menos innovadoras.

El apartado séptimo se dedica a explorar la posibilidad de que las relaciones con los centros de FP tengan una influencia en la participación de los trabajadores en la innovación. Se muestra que las empresas cuyos trabajadores realizan formación continua en los centros de FP presentan mayores niveles de actividad innovadora de todo tipo. Controlando por sector, se constata que estas relaciones son especialmente significativas en los sectores de metalmecánica.

A continuación, al objeto de identificar factores diferencialmente asociados a la participación de los trabajadores, controlando a su vez los efectos múltiples de un conjunto de variables potencialmente explicativas, se presentan los resultados de una regresión logística que muestra que la cooperación externa en innovación (indicativa de un grado superior de actividades innovadoras) y, especialmente, la realización de actividades de formación continua con centros de FP, tienen un efecto multiplicador sobre la probabilidad de participación de los trabajadores en la innovación.

Finalmente, en el apartado octavo se exponen las principales conclusiones de la investigación realizada.

\section{MARCO TEÓRICO}

Partimos de una visión de la innovación como proceso de aprendizaje interactivo interno y externo a la empresa, ligado a sus actividades productivas 
habituales, dentro de un entorno institucional y cultural que condiciona la actividad económica (Lundvall, 1992; Edquist, 1997). Las mejoras graduales 0 incrementales serían el principal tipo de innovación, relacionado con la resolución de problemas, la optimización 0 del desarrollo de las tecnologías habituales, así como a la interacción entre fabricantes y usuarios de sistemas técnicos. En este tipo de aprendizaje prima la interacción y el intercambio de conocimiento tácito entre personas y departamentos dentro de la organización, así como la relación estrecha con un conjunto reducido de agentes externos, generalmente clientes 0 proveedores (Jensen et al., 2007; Lundvall y Lorenz, 2007).

Los estudios recientes realizados desde esta perspectiva han dedicado especial atención al entorno regional de la innovación y a su importancia económica (Braczyk et al., 1996; Storper, 1997; Maskell, 2001; 0ECD, 2001). Se considera que las características institucionales de una región y sus infraestructuras de conocimiento actúan como dinamizadoras de las actividades de empresariales innovación, especialmente en lo que respecta a las pymes (Isaksen y Asheim, 2003). Así, diferentes estudios destacan que la región es especialmente importante para el intercambio de conocimiento tácito y para la provisión de mano de obra cualificada, ambos mecanismos muy importantes en los procesos de innovación de las empresas de menor tamaño y menos intensivas en actividades de I+D formal, que forman la mayor parte del tejido empresarial (Grotz y Braun, 1997; Doloreux, 2003; Asheim y Coenen, 2005; Kauffmann y Tödtling, 2003; Gebauer et al., 2005).

En paralelo, con mayor o menor contacto con la literatura de sistemas regionales de innovación, otro segmento de literatura ha abordado las especificidades de las empresas (especialmente pymes) de sectores de media y baja intensidad tecnológica, que representan una parte muy importante del valor añadido y del empleo en Europa ${ }^{1}$. Distintos estudios han mostrado que las in-

1 Hirsch-Kreisen (2008, p. 12) estimaba que las industrias de media y baja tecnología representaban el $60 \%$ del empleo del sector manufacturero en la UE-15. Frietsch y Neuhaüsler (2015, p. 71) hacen una estimación similar para Alemania. dustrias menos intensivas en I+D son importantes tanto en sí mismas, por su contribución al crecimiento económico, como por su interacción con los sectores de mayor intensidad tecnológica, al implantar las nuevas tecnologías que emanan de estos proveedores tecnológicos y realizar demandas sofisticadas a dichos sectores (Robertson, Smith y von Tunzelmann, 2009; Sandven, Smith y Kaloudis, 2005).

Otras características del modo de innovación low-tech, señaladas por los estudios recientes, serían la mayor importancia relativa de las innovaciones de procesos, que inciden en parámetros como la calidad, el tiempo de respuesta y la productividad, así como una menor colaboración externa en innovación (Kirner, Som y Jäger, 2015, Heidenreich, 2009; Arundel, Bordoy y Kanerva, 2008). También se ha mostrado, con evidencia cualitativa, la especial incidencia en la innovación del personal de producción, incluyendo no sólo ingenieros, sino también técnicos, trabajadores intermedios y operarios (Hirsch-Kreinsen, 2008, 2015).

En esta línea, otros estudios sobre la innovación en sectores de menor intensidad de I+D han mostrado la incidencia de las actividades de formación en la innovación de producto (Barge-Gil, Nieto y Santamaría, 2008) y en el carácter novedoso de la innovación (Amara, Landry, Becheikh y Ouimet, 2008). Este último trabajo señala también la relación entre cooperación externa y novedad de la innovación. Se constata que la mayoría de las pymes low-tech innovan, siendo el reto aumentar el grado de la innovación. En este sentido, Freel (2005) ha indicado que, en este tipo de empresas, lo que marca la diferencia en el grado de novedad de la innovación no es tanto la presencia de personal de ingeniería o científicos, sino el peso de técnicos y trabajadores intermedios cualificados.

En general, los estudios sobre las actividades de formación muestran una incidencia en la productividad, mientras que las relaciones con la innovación han sido mucho menos estudiadas (CEDEFOP, 2011). Además, como indica este informe (ibíd., p. 10), la mayor parte de estudios utilizan mediciones de existencia o no de actividades de formación, o duración de las mismas, sin 
profundizar en los tipos de formación 0 agentes implicados. En el caso español, se ha mostrado la relación entre formación e innovación, aunque referida al nivel directivo (García Espejo, 2008).

Aunque la perspectiva sistémica postula el "enraizamiento" de las actividades de innovación en el "sistema de desarrollo de los recursos humanos" (Lundvall, 2002; Lundvall y Christensen, 2003), esto es, en el sistema educativo y formativo nacional o regional, los estudios recientes se han centrado, en lo que al papel de las infraestructuras de conocimiento se refiere, en el estudio de centros tecnológicos y universidades, descuidando el papel que agentes como los centros de Formación Profesional pudieran tener, sobre todo en relación con las pymes.

El estudio del papel de los institutos técnicos de FP se retrotrae a los estudios sobre clusters y distritos industriales, siendo una referencia inicial a este respecto los trabajos de Stuart Rosenfeld sobre algunas regiones de Estados Unidos (Rosenfeld, 1998a, 1998b). Este autor sugirió que los institutos de educación y formación profesional técnica están en mejor posición que las universidades para dar servicio a las pymes, y que en algunos sectores industriales existe un problema de falta de técnicos, y no tanto de ingenieros (Rosenfeld, 1998a., pp. 6 y 35).

En su informe para la OCDE, este autor (ibíd., pp. 9 y ss.) destaca que los centros de FP realizan distintas funciones en relación con el sistema económico, algunas "tradicionales" como la educación reglada (formación de capital humano), y otras "no tradicionales" como la formación continua de trabajadores y los servicios técnicos a las empresas (pudiendo llegar al apoyo a las pymes en los procesos de innovación). El papel que los institutos técnicos de FP pueden jugar en el desarrollo regional ha sido reconocido en trabajos más recientes (Curtain, 2004; Moodie, 2006; Veluzzi, 2010), aunque como señala este último autor (ibíd., p. 205), dicho papel no ha sido suficientemente estudiado.

Así mismo, diferentes estudios (Whittingham, Ferrier y Trood, 2004; Guthrie y Dawe, 2004) han puesto de manifiesto que la participación de los centros de FP en misiones diferentes de las tradicionales se ve obstaculizada a menudo por diversas barreras como la falta de autonomía 0 flexibilidad de los institutos, la falta de incentivos 0 de instrumentos públicos de fomento y la falta de relación con otros agentes de I+D tales como los centros tecnológicos.

La falta de conexión entre la formación profesional y la innovación se retrotrae, en última instancia, a factores culturales e institucionales. Como han indicado Bosch y Charest (2008, p. 430), mientras que en algunos países (el ejemplo típico sería Alemania) la posesión de una credencial educativa en FP es una señal de que el egresado en cuestión tiene capacidad para realizar autónomamente tareas complejas en un ámbito ocupacional amplio, en otros países dicho título lleva todavía asociada una etiqueta de menor éxito académico y de capacidad de realizar únicamente tareas específicas de baja cualificación. Este sería en gran medida el caso de España, donde la Formación Profesional ha tenido una evolución un tanto "tortuosa", entre los intentos de evitar el estigma del fracaso académico y la evidencia de que el acceso mayoritario a la educación superior general universitaria (incluidos los/as estudiantes más capacitados) ha continuado reproduciendo dicho estigma (Pérez Díaz y Rodríguez, 2002).

Los estudios recientes sobre la situación española, referidos al actual marco normativo, emanado de la Ley Orgánica General del Sistema Educativo (LOGSE) de 1990, insisten en la falta de adaptación de la oferta formativa reglada (incluida la formación práctica) a las necesidades del tejido productivo (Hermosilla Pérez y Ortega, 2003; García Ruiz, 2008; Aguilar Gonzálvez, 2015), así como, en relación con lo anterior, a la necesidad de aumentar las estancias formativas de los profesores en las empresas y la presencia en la docencia de expertos procedentes del ámbito laboral (Jiménez Jiménez, 1997; Field, Kis y Kuczera, 2012).

Una de las principales novedades introducidas por la LOGSE fue la implantación del módulo obligatorio de prácticas de formación en centros de trabajo (FCT), inspirada en el modelo alemán (Pérez Díaz et al., op. cit., p. 186), que parece haber tenido unos efectos positivos como mecanismo 
de selección y contratación de egresados, sobre todo procedentes del ciclo superior (López Mayán y Nicodemo, 2015). Sin embargo, otros autores han indicado que los contenidos prácticos de la LOGSE son más bajos que los de la Ley General de Educación (LGE) de 1970 (Martínez Usarralde, 2000). En este sentido, el extenso estudio de Pérez Díaz y Rodríguez muestra que la LGE supuso, a su vez, una reducción de horas de práctica respecto a la Ley de Formación Profesional Industrial de 1955, que en sus niveles de Oficialía (tres cursos, comenzando a los 14 años) y Maestría Industrial (dos cursos) contaba con un $40-45 \%$ del currículum de carácter práctico (op. cit., p. 108), además de unas denominaciones en clara correspondencia con las categorías laborales existentes².

El debate sobre la falta de adecuación entre la oferta formativa reglada y la demanda empresarial, así como las deficiencias detectadas en la formación para el empleo (ocupacional y continua), han sido el marco de una serie de reformas recientes a partir de la Ley Orgánica de las Cualificaciones y la Formación Profesional (2002), que trata de dar un carácter integrado al sistema formativo. Desde el punto de vista de este artículo, nos interesan principalmente los cambios relativos a las prácticas de FCT y a la formación continua.

En cuanto a las prácticas, la promulgación del Real Decreto 1529/2012 de bases de la formación profesional dual, ha posibilitado una incipiente implantación de este esquema en el ámbito de la FP reglada, todavía pendiente de desarrollo normativo y organizativo por parte de las administraciones educativas. Aunque el decreto responde a un contexto de grave crisis económica y elevado desempleo juvenil, la posibilidad de aumentar la extensión de las prácticas en las empresas (incluida la opción de alargar la formación durante un tercer año), puede verse, al menos en parte, como una res-

2 La elevación, con la LOGSE, de la edad de educación secundaria obligatoria a los 16 años y la eliminación del carácter terminal de la FP1 (heredera de la oficialía) no parece haber contribuido a la disminución del fracaso escolar en educación obligatoria en España, que ronda el $30 \%$. puesta a las insuficiencias detectadas en el currículum práctico de los ciclos formativos.

Algunos estudios subrayan la necesidad de periodos de prácticas más largos para que las empresas, sobre todo pymes, puedan obtener beneficios ( 0 al menos no incurrir en costes) de dichas prácticas (Wolter y Mühlemann, 2015). La implantación de las prácticas en alternancia puede ser un paso más en la dirección del modelo alemán, aunque en un entorno cultural muy diferente, caracterizado por una menor coordinación y regulación entre los agentes implicados, así como un menor nivel de confianza y cooperación en las relaciones laborales. En todo caso, otros estudios indican que el pago al aprendiz de un salario inferior a los beneficios que genera a la empresa durante el periodo de prácticas puede resultar satisfactorio para aquél, siempre que realice tareas cualificadas (las empresas que "condenen" a los aprendices a realizar tareas rutinarias, poco cualificadas, recibirían peores candidatos en el futuro) (Wolter y Ryan, 2011).

Respecto a la formación continua, hay que señalar que, a pesar del incremento en los últimos años de las actividades de formación en las empresas españolas, los agentes implicados (Gobierno y principales organizaciones empresariales y sindicales) reconocen importantes debilidades en el sistema, como son "la deficiente coordinación del conjunto del sistema; la falta de planificación estratégica de la formación profesional para el empleo; su escasa vinculación con la realidad del tejido productivo, especialmente la formación de demanda dirigida a la pyme; la no disponibilidad de un sistema de información integrado; la falta de evaluación de su impacto; y una definición poco eficiente del papel de los agentes implicados en el sistema" (Real Decreto-ley 4/2015, para la reforma urgente del Sistema de Formación Profesional para el Empleo en el ámbito laboral, preámbulo, p. 25019).

Desde la firma a comienzos de los noventa de los primeros Acuerdos Nacionales para la Formación Continua, con el surgimiento de la Formación para la Formación Continua (FORCEM) y más tarde la Fundación Tripartita para 
la Formación en el Empleo (año 2000), Ios principales agentes sindicales y empresariales han dominado el campo de la formación continua (Pérez Díaz y Rodríguez, 2002; Homs, 2008), ejerciendo tanto la formulación como, en gran medida, la implantación de estas políticas (a través de sus propios centros de formación), y dejando muy poco espacio para los centros de FP. Estudios recientes han mostrado que la participación de los centros de FP en la formación para el empleo ha sido mínima, con la excepción de la Comunidad Autónoma del País Vasco (Albizu et al., 2013; Olazaran y Brunet, 2013, cap. 8). El propio preámbulo del Real Decretoley 4/2015 reconoce que sólo a partir de la Ley 3/2012 se "permitió, por primera vez, el acceso de los centros de formación a los fondos disponibles para la gestión de los planes de formación de los trabajadores que hasta la fecha estaban limitados a las organizaciones empresariales y sindicales" (RDL 4/2015, p. 25022). No obstante, también es preciso señalar que Ios centros de FP, sobre todo los públicos, presentan importantes rigideces internas (falta de autonomía de los centros, falta de incentivos al profesorado, límites legales) que deben superarse para un verdadero desarrollo de la figura del centro integrado, que imparta formación reglada y continua con normalidad.

\section{METODOLOGÍA EMPLEADA}

El trabajo realizado ha tenido dos fases, cualitativa y cuantitativa. Para la fase cualitativa se seleccionó un centro de Formación Profesional del área de Pamplona que tiene una larga tradición de colaboración con empresas industriales del entorno. Dentro de este centro se realizaron entrevistas a 5 profesores que cuentan con una larga experiencia en la tutorización de las prácticas de los alumnos en empresas (Formación en centros de trabajo, FCT). Por otro lado, el centro de FP nos facilitó el acceso a 3 empresas donde se realizaron entrevistas a 4 responsables de recursos humanos o instructores de alumnos de la FCT (Directores de producción, jefes de taller).
Las entrevistas cualitativas (semiestructuradas en profundidad) se realizaron entre abril y junio de 2013. Se eligieron empresas de distintos tamaños para ilustrar distintos tipos de participación del personal de FP: dos del sector de fabricación de maquinaria (de 444 y 38 empleados, ambas exportadoras y pertenecientes a grupos empresariales) y una micropyme del sector de fabricación de material y equipo eléctrico (7 empleados, exportadora y no pertenece a un grupo). Todas las empresas pertenecen al sector de metalmecánica, en el cual estamos especialmente interesados en este trabajo por su relación con la FP, y están clasificadas como de nivel tecnológico medio-alto, siendo las dos primeras altamente innovadoras.

El objetivo de la fase cualitativa fue, por un lado, ilustrar aspectos clave de las relaciones entre empresas y centros de FP y de la percepción de las empresas sobre la oferta formativa. Por otro lado, se pretendía ilustrar distintos tipos de participación de los trabajadores con perfil de FP en los procesos de innovación. Todo ello al objeto de preparar la fase cuantitativa de la investigación.

Posteriormente, entre diciembre de 2013 y enero de 2014, se realizó una encuesta telefónica a pequeñas y medianas empresas (pymes) industriales de la Comunidad Foral de Navarra. El estudio cuantitativo se llevó a cabo considerando como población a todas las empresas de entre 10 y 250 empleados/as con actividad en sectores CNAE2009 05-39 (pymes manufactureras) y con sede en la región. El tamaño de muestra global $(\mathrm{N}=219)$ garantiza la representatividad estadística del colectivo de empresas, con un error muestral máximo del $5 \%$ para estimaciones a un NC95, $5 \%$ en el supuesto de diversidad más desfavorable $(p=q=0,5)$. La muestra fue estratificada en tres niveles de tamaño, con selección final aleatoria dentro de cada estrato.

El perfil de las empresas encuestadas corresponde a empresas pequeñas, un $82,2 \%$ tiene menos de 50 empleados, y entre éstas casi la mitad $(43,4 \%)$ tiene menos de 25 empleados, teniendo sólo un $18,8 \%$ de estas pymes 50 más empleados. Se trata de empresas que operan en 
una gran diversidad de sectores, con un importante peso de las actividades relacionadas con la metalmecánica: metalurgia y fabricación de productos metálicos y fabricación de maquinaria y equipo ( $26,0 \%$ y $22,8 \%$ respectivamente). Así mismo las empresas que operan en sectores como la industria de la alimentación, bebidas, tabaco, textil, cuero y calzado, madera y corcho suponen un $29,7 \%$ de la muestra. Por último, el $71,6 \%$ de las empresas se encuadran en sectores de nivel tecnológico bajo (34,2\%) o medio-bajo $(37,4 \%)$, un $22,4 \%$ operan en sectores de nivel tecnológico medio-alto y sólo un $8 \%$ en sectores de alto nivel tecnológico.

Por medio de la encuesta realizada se ha pretendido, por un lado, avanzar en el conocimiento de la percepción que las empresas tienen sobre la oferta formativa, así como en el análisis de las relaciones entre empresas y centros formativos. Por otro lado, se ha realizado una primera aproximación a la medición de la participación del personal de FP en los procesos de innovación de las empresas, considerando distintos tipos de innovación (incluida la mejora de procesos y productos), así como diferencias de tamaño o sector de las empresas.

En particular, se ha intentado averiguar si algún aspecto de las relaciones con los centros formativos puede incidir en una mayor participación de los trabajadores en los procesos de innovación, lo que llevaría, de alguna manera, a reconocer un posible papel de los centros de FP en el sistema de innovación.

\section{PRESENCIA DEL PERSONAL DE FP EN LAS EMPRESAS. PERCEPCIÓN DE LAS EMPRESAS SOBRE LA OFERTA FORMATIVA}

La principal función de los centros educativos es la formación de personal técnico y trabajadores/as cualificados y su inserción en el tejido económico del entorno. En este sentido, la encuesta realizada ha permitido una medición del grado de penetración del personal de FP en las empresas de la región. Así, las pymes encuestadas cuentan con una media de un $38 \%$ de sus plantillas con perfil de FP. El grupo de empresas más numeroso (44\%) es el que tiene plantillas con menos de un $25 \%$ de trabajadores con perfil de FP. Sin embargo, un $31 \%$ de las pymes tiene, al menos, la mitad de su plantilla con este tipo de formación.

Dentro del colectivo de trabajadores de FP, los técnicos superiores suponen el $34 \%$, siendo las situaciones algo más diversas. Por una parte, nos encontramos con un $27 \%$ de pymes en las que nadie posee ese perfil, aunque hasta un $43 \%$ de las empresas analizadas tienen, al menos, la mitad de los trabajadores/as de FP con nivel de formación superior 0 equivalente, hecho que denota un notable grado de cualificación de las plantillas de un determinado grupo de empresas navarras.

Se ha preguntado a las empresas por la capacitación profesional de sus empleados/as al entrar en la empresa. En general, un $71 \%$ de las pymes encuestadas considera que los egresados de FP están bastante o muy capacitados cuando empiezan a trabajar en la empresa, frente a un $29 \%$ de respuestas críticas ("nada-poco capacitados"). No obstante, hay que matizar que las pymes de sectores relacionados con la metalmecánica presentan un porcentaje de respuestas críticas significativamente mayor al del resto de sectores (un 36,4\% considera que los egresados de FP están nada o poco capacitados cuando empiezan a trabajar en la empresa).

Las empresas encuestadas consideran que el personal de FP contribuyen de manera significativa a la competitividad de las mismas. La valoración media es 6,4 en una escala $0-10$ y un $52 \%$ de las empresas otorgan una puntuación entre 7 y 8 puntos. En relación con las características de las empresas, hay que destacar que las pymes de nivel tecnológico medio alto y sobre todo, de sectores ligados a la metalmecánica, dan una puntuación mayor a la contribución que los recursos humanos con FP hacen a la competitividad de la empresa. La valoración también es superior entre las empresas más innovadoras y entre las que tienen relaciones más completas con los centros de FP (alumnado en prácticas, cooperación en proyectos de innovación). 
Tabla 1. Valoración de la contribución de los trabajadores de FP a la competitividad de las empresas según tamaño, sector y nivel tecnológico, capacidad innovadora y grado de relación con centros de FP

\begin{tabular}{l|c}
\hline Tamaño & Media \pm DT \\
\hline Hasta 49 empleados & $6,36 \pm 2,1$ \\
\hline De 50 y más & $6,33 \pm 2,2$ \\
\hline Sector** & \\
\hline Metalmecánica & $6,87 \pm 1,7$ \\
\hline Resto & $5,86 \pm 2,4$ \\
\hline Nivel tecnológico* & \\
\hline Alto y medio-alto & $6,81 \pm 1,4$ \\
\hline Medio bajo y bajo & $6,22 \pm 2,3$ \\
\hline Total & $6,35 \pm 2,1$ \\
\hline Innovadora & \\
\hline Sí & $6,50 \pm 2,1$ \\
\hline No & $5,74 \pm 2,4$ \\
\hline Alumnado FCT** & $6,35 \pm 2,1$ \\
\hline Sí & \\
\hline No & $6,87 \pm 1,7$ \\
\hline Cooperación con centros de formación (Incluidos centrosFP)** & $5,98 \pm 2,3$ \\
\hline Sí & \\
\hline No & $7,52 \pm 1,4$ \\
\hline Total & $6,25 \pm 2,1$ \\
\hline
\end{tabular}

*Significativo NS $<0,05$ ** Significativo NS $<0,01$

Fuente: Elaboración propia

Por otro lado, se ha solicitado a las empresas una valoración sobre la oferta formativa del entorno, así como una comparación con formas anteriores de Formación Profesional. El 62,5\% de las empresas que tienen relaciones con los centros de FP consideran que las enseñanzas regladas que les ofrecen estos centros se adecuan a sus necesidades. No obstante, las empresas muestran un impor- tante grado de desconocimiento sobre las diferencias entre el antiguo (FP1+FP2) y el nuevo sistema de Formación Profesional y sólo un 64\% valora la actual oferta de FP respecto al sistema anterior. De estas empresas, la gran mayoría considera que la oferta actual de grados es mejor. Cabe señalar que se detecta una valoración significativamente más crítica de la oferta formativa de los centros 
de FP entre las empresas de metalmecánica, sector altamente relacionado con estos agentes. Así, alrededor de un cuarto de las empresas de dicho sector considera que las enseñanzas regladas que ofrecen los centros de FP no se adecuan a sus necesidades y/o que la oferta actual de FP no ha mejorado respecto de la antigua Formación Profesional.

Las entrevistas cualitativas realizadas a profesores y empresas del sector de metalmecánica pueden ayudar a interpretar las respuestas obtenidas. En dichas entrevistas nos sorprendió la coincidencia a la hora de apuntar a las limitaciones del actual sistema de Formación Profesional (ciclos de Grado Medio y Superior), proveniente de la LOGSE de 1990. Profesores y empresas señalaron que los ciclos son excesivamente cortos y que se carece de tiempo suficiente tanto para la especialización en contenidos como para la formación práctica en las empresas.

La valoración que las empresas entrevistadas hicieron de la formación teórica del alumnado, por ejemplo en diseño y manufactura asistidos por ordenador, fue en general buena. Se valora la formación generalista, pero se señala que falta una mayor especialización posterior, lo cual exigiría un tercer año en el ciclo de FP superior.

En cuanto al contenido práctico de los ciclos, las valoraciones recogidas fueron muy críticas. La reducción de los ciclos formativos a dos años, con la implantación de la LOGSE, supuso la eliminación de muchas horas de prácticas ("horas de taller") en los centros. Algunas empresas indicaron también que con el sistema de FP anterior (LGE de 1970) Ios alumnos realizaban más horas prácticas en las empresas y de una manera más continuada (unas horas al día después de las clases), mientras que ahora las prácticas se limitan al último trimestre del segundo año del ciclo. Es más, estas empresas apuntaron sin ambages que la FP actual está por debajo incluso de la antigua FP, con su Oficialía y Maestría Industrial (Ley de Formación Profesional Industrial de 1955).

Algunas empresas sugirieron soluciones como alargar el periodo de formación en centros de trabajo, recibir alumnos en distintos momentos del año o conocer desde el inicio de curso los alumnos/as de los que va a disponer, para poder planificar mejor las actividades ${ }^{3}$. En las empresas se señaló la necesidad de reformular las prácticas en base a un mayor seguimiento del trabajo del alumno por parte de la empresa y del centro formativo, así como a una relación más continua entre ambos agentes.

\section{PRÁCTICAS FCT, FORMACIÓN CONTINUA Y SERVICIOS TÉCNICOS}

Tras considerar la presencia del personal de FP en las empresas y la valoración que estas realizan sobre la oferta formativa, pasamos a considerar los distintos tipos de relación que las pymes establecen con los centros formativos.

La mayoría de las empresas encuestadas (58,4\%) manifiesta tener algún tipo de relación con algún centro de FP cercano. Las formas más frecuentes de relación son la recepción de alumnos/as en prácticas de Formación en Centros de Trabajo (FCT, 40,2\% de las empresas) y la contratación de trabajadores a través de las bolsas de trabajo de los centros formativos (31\%).

La siguiente forma de relación según su importancia es la realización de cursos de formación continua, ya sea a la carta, bajo demanda de las empresas (21,5\% de las pymes) o del catálogo que ofrecen los centros (12,8\%). La contratación de servicios técnicos o cesión de maquinaria es menos frecuente ( $9,1 \%$ y $5,0 \%$, respectivamente).

Se detecta que son las empresas más grandes (a partir de 50 empleados), de metalmecánica y/o de nivel tecnológico alto 0 medio alto las que se relacionan en mayor medida con los centros de FP. En las empresas que se encuadran dentro de estos perfiles es significativamente mayor el número de empresas que recibe alumnado en prácticas y contrata a través de las bolsas de trabajo. Además, las empresas más grandes y de nivel tecnológico alto 0 medio alto contratan significativamente más a estos agentes para la realización de actividades de formación continua.

3 Hay que señalar que en el momento de realización de las entrevistas la FP dual no había comenzado a ser implantada en Navarra. 
Tabla 2. Tipos de relaciones que se establecen con centros FP según tamaño, sector y nivel tecnológico

\begin{tabular}{l|c|c|c|c|c|c|c}
\hline & \multicolumn{2}{|c|}{ Tamaño } & \multicolumn{2}{c|}{ Nivel tecnológico } & \multicolumn{2}{c|}{ Sector } & \multirow{2}{*}{ TOTAL } \\
\cline { 2 - 7 } & Hasta $\mathbf{5 0}$ & $\mathbf{5 0}$ y más & $\begin{array}{c}\text { Alto 0 } \\
\text { medio alto }\end{array}$ & $\begin{array}{c}\text { Medio bajo } \\
\text { 0 bajo }\end{array}$ & Metal & Resto & \\
\hline Algún tipo de relación & 55,0 & $74,4^{*}$ & $75,5^{*}$ & 52,9 & $68,2^{*}$ & 49,1 & 58,4 \\
\hline Alumnos en práctica & 37,2 & $64,1^{*}$ & $62,3^{*}$ & 35,0 & $52,3^{*}$ & 32,1 & 42,0 \\
\hline Bolsas de trabajo & 27,2 & $46,2^{*}$ & $47,2^{*}$ & 25,5 & $44,9^{* *}$ & 17,0 & 30,6 \\
\hline Formación continua & 26,1 & $43,6^{*}$ & $39,6^{*}$ & 25,5 & 32,7 & 25,9 & 29,2 \\
\hline Servicios técnicos & 8,9 & 10,3 & 7,5 & 9,6 & 10,3 & 8,0 & 9,1 \\
\hline Cesión maquinaria & 4,4 & 7,7 & 5,7 & 3,8 & 5,6 & 4,5 & 5,0 \\
\hline$N$ & 180 & 39 & 53 & 157 & 107 & 112 & 219 \\
\hline
\end{tabular}

* Significativo NS $<0,05$

** Significativo NS $<0,01$

Fuente: Elaboración propia

Es interesante destacar el papel que las prácticas FCT pueden estar jugando como forma básica de relación entre centros formativos y empresas, a partir de la cual pueden establecerse relaciones más "avanzadas" —o menos comunes - como pueden ser la formación continua o la prestación de servicios técnicos. Así, el hecho de tener alumnado en prácticas está fuertemente relacionado con la asistencia a cursos de formación continua impartidos por los centros, tanto de oferta como de demanda. A este respecto hay que indicar que alrededor de un $80 \%$ de las empresas muestran una actitud favorable a la posibilidad de que los centros de FP amplíen su oferta de formación continua y/0 servicios de apoyo a la innovación.

Tabla 3. Tipos de relaciones que se establecen con centros FP según existencia de alumnos en prácticas FC

\begin{tabular}{|c|c|c|c|c|c|c|}
\hline & \multicolumn{4}{|c|}{ Alumnado en prácticas FCT } & \multirow{2}{*}{\multicolumn{2}{|c|}{ Total }} \\
\hline & \multicolumn{2}{|c|}{ sí } & \multicolumn{2}{|c|}{ NO } & & \\
\hline & N & $\%$ & N & $\%$ & $\mathbf{N}$ & $\%$ \\
\hline Bolsas de trabajo** & 57 & 62,0 & 10 & 7,9 & 67 & 30,6 \\
\hline Formación continua de oferta** & 21 & 22,8 & 7 & 5,5 & 28 & 12,8 \\
\hline Formación continua a la carta** & 29 & 31,5 & 18 & 14,2 & 47 & 21,5 \\
\hline Servicios técnicos & 12 & 13,0 & 8 & 6,3 & 20 & 9,1 \\
\hline Cesión maquinaria & 7 & 7,6 & 4 & 3,1 & 11 & 5,0 \\
\hline $\mathrm{N}$ & \multicolumn{2}{|c|}{92} & \multicolumn{2}{|c|}{127} & \multicolumn{2}{|c|}{219} \\
\hline
\end{tabular}

** Significativo NS $<0,01$

Fuente: Elaboración propia 
En general, la evidencia cuantitativa recogida muestra la importancia de las prácticas FCT como forma de relación entre la oferta formativa y el tejido empresarial y como mecanismo de selección de personal cualificado por parte de las empresas. También se confirma que, tras la formación reglada de capital humano y su inserción en la empresa, la provisión de formación continua constituye el principal servicio que los centros formativos pueden ofrecer al entorno económico. En este sentido, a partir de las entrevistas cualitativas realizadas emergen algunas cuestiones que pasamos a considerar.

Respecto a la cultura formativa, en las entrevistas realizadas surgió una distinción entre empresas innovadoras, que creen en su personal y fomentan la formación en sus distintas facetas (interna, con proveedores tecnológicos y con centros formativos) y empresas más "cortoplacistas", generalmente más pequeñas, que temen que, si sus trabajadores reciben formación, puedan abandonar la empresa. En las empresas más grandes se apuntan dos tipos de actitudes hacia la formación por parte de los trabajadores: una más receptiva, por parte de personal de áreas como Oficina Técnica, y otra menos receptiva en personal de montaje.

Los profesores entrevistados señalaron las limitaciones al desarrollo de la oferta de formación continua desde los centros de FP debidas a falta de aportación de fondos por parte de los agentes sociales y del Departamento de Empleo del gobierno regional, así como a la rigidez organizativa de los centros formativos públicos.

Desde las empresas se recogieron algunos testimonios críticos hacia la falta de implicación de los profesores de los centros en la formación continua, que provoca una falta de optimización en el uso de recursos formativos de los centros (por ej. autómatas programables) que serían muy interesantes para las empresas. En todo caso, en general, de las entrevistas realizadas se desprende que, para que la formación continua ofrecida por los centros de FP interese a las empresas, debe ser especializada, esto es, deben ser cursos específicos que vayan más allá de la formación de oferta generalista.
El equipamiento de los centros formativos pueden ser también utilizado para dar servicios técnicos a las empresas, tal y como se documentó en un caso exitoso detectado en el centro formativo entrevistado (colaboración en la automatización de procesos de trabajo manual de soldadura). Se trata de casos puntuales que pueden ser reforzados con estancias de los profesores en las empresas y que muestran un potencial de los centros formativos insuficientemente explotado hasta el momento.

\section{INNOVACIÓN Y PARTICIPACIÓN DEL PERSONAL DE FP}

La realización de actividades vinculadas a la innovación está muy extendida entre las empresas navarras encuestadas. En este trabajo se ha considerado un concepto amplio de innovación que incluye las mejoras de producto y proceso, y que consideramos que es especialmente interesante en un entorno como el español, en el que predominan las pymes y los sectores de baja y media intensidad tecnológica. El abanico de actividades documentadas va desde la innovación de producto (69\% de las pymes), como la más específica, hasta la mejora de procesos (91\%), que aparece como la forma de innovar más extendida. No se aprecian diferencias significativas en función del tamaño, sector de actividad o el nivel tecnológico de las empresas. A este respecto, es interesante comentar que las empresas parecen innovar independientemente del sector de intensidad tecnológica al que están estadísticamente adscritas, tal y como se señala desde la literatura sobre innovación en sectores low-tech. También se confirma que las innovaciones más importantes en dichos sectores son las relacionadas con los procesos productivos. Sin embargo, en contra de lo que indican algunos trabajos desarrollados desde dicha perspectiva, no se observan diferencias en cuanto a innovación en producto, esto es, empresas de distintos niveles de intensidad tecnológica tienen un nivel similar de desarroIlo de nuevos productos. 
Tabla 4. Empresas en las que se realizan las siguientes actividades de innovación (\%) según tamaño, sector y nivel tecnológico

\begin{tabular}{l|c|c|c|c|c|c|c}
\hline & \multicolumn{2}{|c|}{ Tamaño } & \multicolumn{2}{c|}{ Sector } & \multicolumn{2}{c}{ Nivel tecnológico } & Total \\
\cline { 2 - 8 } & Hasta 50 & $\mathbf{5 0}$ o más & Metal & Resto & Alto & Bajo & \\
\hline Innovación producto & 68,9 & 71,8 & 65,4 & 73,2 & 71,7 & 69,4 & 69,4 \\
\hline Innovación proceso & 78,3 & 87,2 & 78,5 & 81,3 & 81,1 & 79,0 & 79,9 \\
\hline Mejora productos & 81,7 & 87,2 & 78,5 & 86,6 & 83,0 & 83,4 & 82,6 \\
\hline Mejora procesos & 90,6 & 92,3 & 92,5 & 89,3 & 90,6 & 91,1 & 90,9 \\
\hline $\begin{array}{l}\text { Nuevos sistemas } \\
\text { organización }\end{array}$ & 85,0 & 89,7 & 86,0 & 85,7 & 84,9 & 86,6 & 85,8 \\
\hline Nuevos mercados & 85,6 & 84,6 & 85,0 & 85,7 & 83,0 & 86,6 & 85,4 \\
\hline $\mathbf{N}$ & 180 & 39 & 107 & 112 & 53 & 157 & 219 \\
\hline
\end{tabular}

Fuente: Elaboración propia

Uno de los objetivos centrales de la investigación realizada era la medición de participación del personal de FP en las actividades de innovación. A este respecto, hay que señalar que dicha participación es relevante, especialmente en las actividades relacionadas con la mejora de procesos y productos. Así un $73,4 \%$ y $60,8 \%$, respectivamente, de pymes declara que los trabajadores de FP tienen una participación importante en dichas activida- des. Por otro lado, en más de la mitad de las pymes encuestadas (52\%) el personal de FP participa en actividades de innovación de procesos. De manera secundaria, también nos encontramos con porcentajes reseñables de participación en innovaciones organizativas $(44,1 \%)$ e innovación de producto $(38,8 \%)$. No se detectan diferencias significativas de participación en función del tamaño, sector 0 nivel tecnológico de las empresas.

Tabla 5. Empresas en las que los trabajadores participan en actividades de innovación (\%) según tamaño, sector y nivel tecnológico

\begin{tabular}{l|c|c|c|c|c|c|c}
\hline & \multicolumn{2}{|c|}{ Tamaño } & \multicolumn{2}{c|}{ Sector } & \multicolumn{2}{c}{ Nivel tecnológico } & \multirow{2}{*}{ Total } \\
\cline { 2 - 7 } & Hasta $\mathbf{5 0}$ & $\mathbf{5 0} \mathbf{0}$ más & Metal & Resto & Alto & Bajo & \\
\hline Innovación producto & 39,5 & 35,7 & 42,9 & 35,4 & 36,8 & 38,5 & 38,8 \\
\hline Innovación proceso & 50,4 & 58,8 & 51,2 & 52,7 & 46,5 & 53,2 & 52,0 \\
\hline Mejora productos & 60,5 & 61,8 & 64,3 & 57,7 & 63,6 & 59,5 & 60,8 \\
\hline Mejora procesos & 73,0 & 75,0 & 71,7 & 75,0 & 77,1 & 71,3 & 73,4 \\
\hline $\begin{array}{l}\text { Nuevos sistemas } \\
\text { organización }\end{array}$ & 42,5 & 51,4 & 43,5 & 44,0 & 44,4 & 44,9 & 4 \\
\hline Nuevos mercados & 20,8 & 33,3 & 22,0 & 24,0 & 27,3 & 20,6 & 23,0 \\
\hline
\end{tabular}


En cambio, sí se encuentran diferencias en la participación de estos trabajadores según el carácter innovador de las empresas. Así, entre las empresas que se consideran más innovadoras que sus competidores directos, independientemente del sector de intensidad tecnológica en el que estén oficialmente clasificadas, la participación de los trabajadores de FP en actividades relacionadas con la innovación es mayor en todas las actividades consideradas, especialmente en innovaciones y mejoras de producto, así como en innovaciones de proceso, esto es, en el conjunto de innovaciones que tienen un mayor grado de novedad. De este modo, las pymes más innovadoras desarrollan un nivel de innovaciones de producto y proceso significativamente mayor $(83,1 \%$ y $97,2 \%$, respectivamente) que las pymes menos innovadoras (69,9\% y $79,7 \%$ ).

La evidencia recogida en las entrevistas cualitativas a empresas puede ayudar a ilustrar algunos tipos principales de participación del personal de FP en las actividades de innovación. Un primer aspecto que destaca en las entrevistas realizadas es la importancia de la actitud de las personas, su integración en la organización, su iniciativa y sus ganas de aprender, de cara a la participación en los procesos de innovación. Se trata, como indica una empresa, de "cambiar el chip de que la empresa me tiene que dar todo a que yo me tengo que buscar también la vida".

Sistematizando la información recogida, podemos hablar de tres niveles de participación. El primer tipo de participación, más básico, referido a grupos 0 iniciativas de mejora, correspondería a operarios de menor cualificación, generalmente encargados de tareas más rutinarias. A este nivel, las empresas señalan la importancia de aprender de los problemas y averías que se presentan en las actividades productivas habituales y de que los trabajadores sepan trabajar en las distintas máquinas de la empresa. Para que esta polivalencia sea posible, son necesarios trabajadores con mayor formación capaces de manejar maquinaria sofisticada, especialmente de control numérico. Cuanto menor es la cualificación de los trabajadores y mayor su edad, existe una mayor probabilidad de actitudes reacias al cambio.

Tabla 6. Participación de los trabajadores de FP en los distintos tipos de innovación según la capacidad comparativa de innovación de las empresas

\begin{tabular}{|c|c|c|c|c|c|c|c|}
\hline & & \multicolumn{4}{|c|}{ Capacidad comparativa innovación } & \multirow{2}{*}{\multicolumn{2}{|c|}{ Total }} \\
\hline \multirow{2}{*}{\multicolumn{2}{|c|}{ Participan }} & \multicolumn{2}{|c|}{ Innovadora } & \multicolumn{2}{|c|}{ No innovadora } & & \\
\hline & & $\mathbf{N}$ & $\%$ & $\mathrm{~N}$ & $\%$ & $\mathbf{N}$ & $\%$ \\
\hline \multirow{2}{*}{ Innovación producto* } & Nada/Poco & 38 & 64,4 & 76 & 81,7 & 114 & 75,0 \\
\hline & Bastante/Mucho & 21 & 35,6 & 17 & 18,3 & 38 & 25,0 \\
\hline \multirow{2}{*}{ Innovación proceso** } & Nada/Poco & 40 & 58,0 & 83 & 78,3 & 123 & 70,3 \\
\hline & Bastante/Mucho & 29 & 42,0 & 23 & 21,7 & 52 & 29,7 \\
\hline \multirow{2}{*}{ Mejora productos** } & Nada/Poco & 36 & 55,4 & 86 & 74,1 & 122 & 67,4 \\
\hline & Bastante/Mucho & 29 & 44,6 & 30 & 25,9 & 59 & 32,6 \\
\hline \multirow{2}{*}{ Mejora procesos } & Nada/Poco & 31 & 43,7 & 72 & 56,3 & 103 & 51,8 \\
\hline & Bastante/Mucho & 40 & 56,3 & 56 & 43,8 & 96 & 48,2 \\
\hline \multirow{2}{*}{$\begin{array}{l}\text { Nuevos sistemas } \\
\text { organización }\end{array}$} & Nada/Poco & 49 & 73,1 & 96 & 79,3 & 145 & 77,1 \\
\hline & Bastante/Mucho & 18 & 26,9 & 25 & 20,7 & 43 & 22,9 \\
\hline \multirow{2}{*}{ Nuevos mercados } & Nada/Poco & 59 & 85,5 & 105 & 89,0 & 164 & 87,7 \\
\hline & Bastante/Mucho & 10 & 14,5 & 13 & 11,0 & 23 & 12,3 \\
\hline
\end{tabular}

\footnotetext{
* Significativo NS $<0,05 / * *$ Significativo NS $<0,01$
} 
La intervención de los técnicos de FP en las actividades de innovación, industrialización 0 mejora de los procesos de fabricación constituye un segundo nivel, más intensivo, de participación en la innovación. La innovación de producto lleva consigo el desarrollo de nuevos procesos productivos, ámbito en el cual las empresas entrevistadas documentan una participación importante de los trabajadores cualificados con perfil de FP. Típicamente, en dichas empresas se da una división entre el diseño o conceptualización de productos/procesos, por un lado, y la implementación de dicho producto o proceso, es decir, su industrialización y plasmación en un nuevo proceso de fabricación, por otro.

Normalmente, las tareas de diseño o conceptualización, en tanto que implican un mayor desarrollo matemático o comprensión teórica (visión espacial, razonamiento), son llevadas a cabo por ingenieros, mientras que las tareas de implementación de los nuevos prototipos y procesos son realizadas en buena medida por trabajadores de FP. En esta segunda fase se realizan bocetos, pruebas, modificaciones 0 inputs basados en la experiencia y conocimiento tácito de los trabajadores de FP que pueden tener una importancia crítica para la puesta en marcha de los nuevos procesos e innovaciones.

En un tercer nivel de participación, mucho menos común, se documentan casos de trabajadores de FP superior con experiencia en la empresa que han continuado su formación en nuevas tecnologías de fabricación y que llegan a participar en tareas de diseño y conceptualización de innovaciones, así como a ocupar puestos de ingeniero técnico 0 jefe de área o departamento. La actitud y la iniciativa de estas personas son, junto a los procesos de formación, un ingrediente central en los técnicos que promocionan a puestos de responsabilidad en las empresas.

Las personas provenientes de la FP superior que amplían su formación en software o realizan estudios posteriores de ingeniería técnica son muy valoradas por las empresas, por tener un "enfoque industrial" del que carecen algunos ingenieros. Se valora mucho la posibilidad de realizar nuevos diseños partiendo del conocimiento de las máquinas y su programación. Como indica un profesor entrevistado, "puede hacer diseño, pero además, cuando hace el diseño, sabe fabricar, porque antes ya ha fabricado" 4 .

\section{LA FORMACIÓN CONTINUA COMO PALANCA DE LA INNOVACIÓN}

Un objetivo central de la investigación realizada era comprobar si las relaciones con los centros de FP pueden incidir en una mayor participación de los trabajadores en la innovación, lo cual supondría que los centros formativos pueden tener un papel en el sistema de innovación que no ha sido explícitamente (o suficientemente) reconocido en la literatura y en las políticas recientes.

En esta línea, los resultados obtenidos han permitido detectar diferencias significativas en los niveles de participación dependiendo de la prestación de servicios de formación continua por parte de los centros de FP. Así, en las empresas donde los empleados realizan cursos de formación continua (de oferta 0 demanda), la participación de los trabajadores de FP en actividades de innovación es significativamente mayor que entre las que no acuden a estos centros. Esta relación es significativa para todos los tipos de actividades de mejora e innovación contempladas: innovación producto, innovación de proceso, mejora de productos, mejora de procesos, introducción de nuevos sistemas organización y búsqueda de nuevos mercados.

Anteriormente se ha resaltado la intensidad de las relaciones que se establecen entre las empresas del sector de metalmecánica y los centros de FP en la región. En la tabla 8 se puede observar el resultado de controlar los posibles efectos de la variable sector de las empresas en esta

4 En palabras de una empresa: "Apostar por un cambio de diseño mecánico, sabiendo que eres capaz de programarlo, le da un valor importantísimo; entonces, ese técnico que domina la programación y al que enseñamos a entender la mecánica y a entender el proceso, la tecnología, tiene un valor incalculable para nosotros. Y hay mucho personal de Formación Profesional ahí". 
relación entre la realización de formación continua con centros de FP y una mayor participación de trabajadores con perfil de FP en actividades de innovación. Así, se detecta que, tanto entre las empresas del sector metal como de otros sectores, hay un mayor porcentaje de empresas en las que los trabajadores participan en actividades de innovación entre aquellas que realizan formación continua con centros de FP. No obstante, las diferencias en participación entre las empresas que tienen relación con centros de FP frente a las que no tienen son mayores entre las empresas del sector metalmecánica, llegando a ser estadísticamente significativas.

Tabla 7. Empresas en las que los trabajadores participan en actividades de innovación (\%) según realización de cursos de formación continua con centros de FP

\begin{tabular}{l|c|c|c}
\hline & $\begin{array}{c}\text { Realizan } \\
\text { formación continua }\end{array}$ & $\begin{array}{c}\text { No realizan } \\
\text { formación continua }\end{array}$ & Total \\
\hline Innovación producto* & 52,3 & 33,3 & 38,8 \\
\hline Innovación proceso* & 67,3 & 45,5 & 52,0 \\
\hline Mejora productos* & 73,7 & 54,8 & 60,8 \\
\hline Mejora procesos* & 87,3 & 66,9 & 73,4 \\
\hline Nuevos sistemas & 70,0 & 32,0 & 44,1 \\
\hline organización** & 36,8 & 16,9 & 23,0 \\
\hline Nuevos mercados* & & &
\end{tabular}

* Significativo NS $<0,05 /$ ** Significativo NS $<0,01$

Fuente: Elaboración propia

Tabla 8. Empresas en las que los trabajadores participan en actividades de innovación (\%) según si realizan cursos de formación continua con centros de FP. Control por sector de actividad

\begin{tabular}{l|c|c|c|c|c|c}
\hline & \multicolumn{3}{|c|}{ METAL } & \multicolumn{3}{c}{ RESTO } \\
\hline & Sí FC & No FC & Total & Sí FC & No FC & Total \\
\hline Innovación producto & $62,5^{*}$ & 32,6 & 42,9 & 40,0 & 33,9 & 35,4 \\
\hline Innovación proceso & $77,8^{*}$ & 38,6 & 51,2 & 56,0 & 51,5 & 52,7 \\
\hline Mejora productos & $82,8^{*}$ & 54,5 & 64,3 & 64,3 & 55,1 & 57,7 \\
\hline Mejora procesos & $97,1^{* *}$ & 57,8 & 71,7 & 75,0 & 75,0 & 75,0 \\
\hline $\begin{array}{l}\text { Nuevos sistemas } \\
\text { organización }\end{array}$ & $78,1^{* *}$ & 25,0 & 56,5 & 60,7 & 38,2 & 44,8 \\
\hline \begin{tabular}{l} 
Nuevos mercados \\
\hline
\end{tabular} & $35,7^{*}$ & 13,3 & 22,0 & 34,6 & 20,0 & 24,0 \\
\hline
\end{tabular}

* Significativo NS $<0,05 / * *$ Significativo NS $<0,01$

Fuente: Elaboración propia 
Tabla 9. Regresión logística. Variable dependiente: participación de los trabajadores en innovación. Modelos de Regresión logística binaria. Predictores de participación (Menor/Mayor)

\begin{tabular}{l|r|r|r}
\hline & \multicolumn{3}{|c}{$\mathrm{N}=97$} \\
\hline Participación (Menor/Mayor) & $\mathrm{B}$ & Wald sig. & Exp(B) \\
\hline Tamaño (pequeño<50) & & & \\
\hline Sector (metal) &, 376 &, 464 & 1,456 \\
\hline Nivel tecnológico (bajo o medio bajo) &, 012 &, 979 & 1,012 \\
\hline Capacidad comparativa de innovación (mayor) &, 350 &, 470 &, 705 \\
\hline Cooperación con agentes externos (Sí) &,- 209 &, 642 &, 811 \\
\hline Relación con centros de FP en formación continua &, 892 &, 038 & 2,440 \\
\hline Constante & 1,674 &, 009 & 5,331 \\
\hline & 0,205 & 0,772 & 1,228 \\
\hline Chi2 sig & & & \\
\hline R2 Nagelkerke & & 0,011 & \\
\hline Prueba Hosmer y Lemeshow sig & & 0,135 & \\
\hline
\end{tabular}

Fuente: Elaboración propia

Al objeto de identificar factores diferencialmente asociados al hecho de que las pymes tengan más participación de trabajadores FP en innovación, se ha realizado una regresión logística binaria tomando como variable dependiente dicha participación y controlando a la vez los efectos múltiples de un conjunto devariables potencialmente explicativas de la participación. De este modo, aparece una combinación de dos tipos de variables como predictoras significativas de la participación de los trabajadores de FP en innovación: por un lado, ser una empresa que coopere con agentes externos en procesos de innovación (lo que indicaría un nivel superior de actividades de innovación) y, sobre todo, realizar formación continua con centros de FP. La cooperación externa en proyectos de innovación duplica la probabilidad de participación, mientras que realizar formación continua con los centros formativos quintuplica dicha probabilidad.

\section{CONCLUSIONES}

A pesar de la expansión en décadas recientes de los estudios de formación profesional reglada y de las actividades de formación continua en
España, existen importantes dudas y debates sobre el equilibrio entre la enseñanza profesional reglada y la enseñanza general académica, sobre el currículum de la FP reglada, sobre la coordinación de sistema de formación en su conjunto y sobre el desarrollo de la figura del centro integrado de FP y su participación en la formación continua.

Partiendo de la literatura reciente sobre la aportación de los centros de FP al desarrollo económico regional, este trabajo ha analizado la situación en la Comunidad Foral de Navarra, una región industrial que presenta importantes niveles de educación profesional y de relación de la misma con el tejido productivo. Se ha estudiado el papel de los trabajadores/as de FP en la empresa y, en particular, su participación en los procesos de innovación. A partir de un concepto amplio de innovación, especialmente adecuado para pymes, así como para sectores de menor intensidad tecnológica, se ha mostrado que el personal de FP tiene un importante nivel de participación en la misma, especialmente en las empresas más innovadoras.

Posteriormente, se ha tratado de averiguar si las relaciones que las empresas establecen con los 
centros de FP pueden llegar a incidir en los procesos de innovación, a través de una mayor participación de los trabajadores cualificados en los mismos. A este respecto, en base a una regresión logística, se ha mostrado que la cooperación con agentes externos en proyectos de innovación (indicadora de un grado mayor de innovación) y, sobre todo, la relación con los centros de FP en servicios de formación continua, ejercen un efecto multiplicador sobre la probabilidad de participación de los trabajadores en los procesos de innovación.

Los resultados obtenidos invitan a una urgente reconsideración del papel secundario que los centros de FP han jugado en el ámbito de la formación continua, así como del insuficiente grado de desarrollo de los centros integrales. También es preciso reflexionar sobre las relaciones entre los centros de FP terciaria y la universidad ${ }^{5}$, así como entre dichos centros y otros agentes del sistema de innovación. Todo ello sin olvidar la importancia de la rama de FP postsecundaria de cara a la reducción del fracaso escolar y del elevado nivel de desempleo juvenil, aspectos que no han sido objeto de estudio en este trabajo.

Finalmente, el énfasis en la necesidad de "cambiar el modelo productivo", recurrente en el debate político, no debe hacer olvidar la importancia de los sectores de menor intensidad tecnológica, mayoritarios en la estructura productiva española, y cuyo nivel innovador se encuentra entre los más bajos de Europa (Robertson y Patel, 2007, p. 716) ${ }^{6}$. La investigación realizada ha mostrado que la participación de los trabajadores/as con perfil de FP, espoleada por las actividades de formación continua, puede ser un ingrediente fundamental del modo de innovación de las pymes industriales y, por tanto, de su supervivencia frente a una competencia creciente de países con menores costes laborales y menor calidad del trabajo.

5 La ubicación de los centros de FP terciaria en institutos de educación postsecundaria es un tanto anómala en el contexto europeo (Field et al., 2012).

6 Según estos autores, en el sector de intensidad tecnológica media-baja España se encuentra en el puesto 14 de 14 países considerados, mientras que en intensidad baja se encuentra en el 13 .

\section{AGRADECIMIENTOS Y FINANCIACIÓN}

Este trabajo ha sido realizado dentro del proyecto "Formación profesional y sistema de innovación: El papel de los trabajadores intermedios en los procesos de innovación de las pymes industriales", financiado por el Ministerio de Ciencia e Innovación, Gobierno de España (CS02011-29410-C03-01). Así mismo, en las fases preliminares y actividades complementarias, el equipo contó con la ayuda a grupos de investigación IT593-13 del Departamento de Educación, Universidades e Investigación del Gobierno Vasco.

\section{REFERENCIAS BIBLIOGRÁFICAS}

Aguilar Gonzálvez, M. (2015). El sistema "alternativo" de formación profesional dual para el empleo en España: perspectivas de evolución. $R e-$ vista Internacional y Comparada de Relaciones Laborales y Derecho del Empleo, 3(4), 1-26.

Albizu, E., Olazaran, M., Lavía, C., Otero, B. (2013). Comunidad Autónoma del País Vasco. En M. Olazaran, I. Bruner, Entorno regional y Formación Profesional: Los casos de Aragón, Asturias, Cataluña, Madrid, Navarra y País Vasco (pp. 189-217). Tarragona: URV Publicacions.

Amara, N., Landry, R., Becheikh, N., Ouimet, M. (2008). Learning and novelty of innovation in established manufacturing SMEs. Technovation, 28, 450-463.

Arundel, A., Bordoy, C., Kanerva, M. (2008). Neglected innovators: How do innovative firms that do not perform R\&D innovate? Manchester: MERIT, Inno-Metrics Thematic Paper.

Asheim, B., Coenen, L. (2005). Knowledge bases and regional innovation systems: comparing nordic clusters. Research Policy, 34(8), 173-190.

Barge-Gil, A., Nieto, M., \& Santamaría, L. (2008). Hidden innovators: The role of non $R \& D$ activities. Copenhagen: Druid Conference.

Bosch, G., \& Charest, J. (2008). Vocational training and the labour market in liberal and coordinated economies. Industrial Relations Journal, 39(5), 428-447.

Braczyk, H., Cooke, P., \& Heidenreich, R. (1996). Regional Innovation Systems: The Role of Go- 
vernances in a Globalized World. London: University College Press.

CEDEFOP. (2010). La modernización de la formación profesional. Luxemburgo: Oficina de Publicaciones de la Unión Europea, <http://www.cedefop. europa.eu/EN/Files/9013_es.pdf> (disponible en febrero de 2011).

CEDEFOP. (2011). The impact of vocational education and training on company performance. $C E$ DEFOP Research Paper No. 19, Luxembourg.

CEDEFOP. (2013). On the way to 2020: Data for vocational education and training policies. Luxembourg: Publications Office of the EU, European Centre for the Development of Vocational Training.

Crespo, J., Sanz, I. (2000). La formación continua en España: implicaciones de política económica. Papeles de Economía Española, 86, 280-294.

Curtain, R. (2004). Innovation nand vocational education and training. En S. Dawe, Vocational Education and Training and Innovation: Research Readings (pp. 42-58). Adelaida, Australia: national Centre for Vocational Education research (NCVER).

Doloreux, D. (2003). Regional innovation systems in the periphery: The case of Beauce in Quebec (Canada). International Journal of Innovation Management, 7(1), 67-94.

Edquist, C. (1997). Systems of Innovation: technologies, Institutions and Organizations. London: Pinter.

Field, S., Kis, V., Kuczera, M. (2012). A skills beyond school commentary on Spain. Paris: OECD Reviews of Vocational Educaiton and Training.

García Espejo, I. (2008). Formación e innovación en las empresas industriales. Papers, 8, 179-194.

García Ruiz, M. (2008). Convergencias y divergencias entre sistema productivo y sistema educativo. Revista Complutense de Educación, 19(1), 209-223.

Gebauer, A., Woon Nam, C., Parsche, R. (2005). Regional technology policy and factors shaping local innovation networks in small German cities. European Planning Studies, 13(5), 661-683.

Grotz, R., Braun, B. (1997). Territorial or transnational networking: Spacial aspects of technologyoriented cooperation within the German mecha- nical engineering industry. Regional Studies, 31(6), 545-557

Gutrie, H., Dawe, S. (2004). Overview. En S. Dawe, Vocational Education and Training and Innovation: Research Readings (pp. 10-19). Adelaida, Australia: NCVER.

Heidenreich, M. (2009). Innovation patterns and location of European low-and medium-technology industries. Research Policy, 38, 483-494.

Hemosilla Pérez, A., Ortega, N. (2003). Formación y organización flexible del trabajo en las empresas europeas del sector metalúrgico: los casos de España, Francia, Italia y Portugal. Revista Europea de Formación Profesional, 28, 53-70.

Hirsch-Kreinsen, H. (2008). "Low technology": A forgotten sector in innovation policy. Journal of Technology Management and Innovation, vol. 3, 11-19.

Hirsch-Kreinsen, H. (2015). Innovation in low-tech industries: Current conditions and future prospects. En 0. Som, E. Kirner, Low-tech Innovation (pp. 17-32). Switzerland: Springer International Publishing.

Homs, 0. (2008). La Formación Profesional en España. Hacia la Sociedad del Conocimiento. Barcelona: Fundación La Caixa.

Isaksen, B., Asheim, A. (2003). SMEs and the regional dimension of innovation. En B. Asheim, A. Isaksen, C. Nauwelaers, y F. Tödtling, Regional Innovation Policy for Small-Medium Enterprises (pp. 21-46). Cheltenham: Edward Elgar.

Jensen, M., Johnson, B., Lorenz, E., Lundvall, B. (2007). Forms of knowledge and modes of innovation. Research Policy, 36(5), 680-693.

Jiménez Jiménez, B. (1997). Formación profesional: Concepción de los ciclos formativos. Revista Interuniversitaria de Formación del Profesorado, 30, 31-49.

Kaufmann, A., Tödtling, F. (2003). Innovation patterns of SMEs. En B. Asheim, A. Isaksen, C. Nauwelaers, F. Tödtling, Regional Innovation Policy for Small-Medium Enterprises (pp. 78-115). Cheltenham: Edward Elgar.

Kirner, E., Som, 0., Jäger, A. (2015b). Innovation strategies and patterns of non-R\&D-performing and non-R\&D-intensive firms. En 0. Som, E. Kirner, Low-tech Innovation (pp. 91-112). Switzerland: Springer International Publishing. 
López Mayan, C., Nicodemo, C. (2015). The transition from vocational education to work: Evidence from Spain. Revista de Economía Aplicada, 67, 93-130.

Lundvall, B. (1992). National Systems of Innovation: Towards a Theory of Innovation and Interactive Learning. London: Pinter.

Lundvall, B. (2002). Innovation, Growth and Social Cohesion: The Danish Model. Cheltenham: Edward Elgar.

Lundvall, B., Christensen, J. (2003). Broadening the analysis of innovation systems: Competition, organisational change and employment dynamics in the Danish system. En P. Conceiçao, M. Heitor, B. Lundvall, Innovation, Learning and Social Cohesion in Europe: Towards a Learning Society (pp. 144-179). Cheltenham: Edward Elgar.

Lundvall, B., Lorenz, E. (2007). Modes of innovation and knowledge taxonomies in the learning economies. Oslo: CAS workshop on innovation in firms.

Manskell, P. (2001). Social capital, innovation and competitiveness. En S. Baron, J. Field, y T. Schuller, Social Capital: Critical Perspectives (pp. 111-123). 0xford: Oxford University Press.

Martínez Usarralde, M. (2000). Reflexiones acerca de la alternancia: una comparación entre el modelo de prácticas a través del módulo FVT y el aprendizaje en España. Contextos Educativos, 3, 297-314.

Ministerio de Empleo y Seguridad Social. (2013). Encuesta de Formación Profesional para el Empleo en Empresas 2010. Síntesis de Resultados. Madrid: Ministerio de Empleo y Seguridad Social, http://www. empleo.gob.es/estadisticas/efpc/EFPCSINT2010/ Sintesis2010.pdf, acceso 25-4-2016.

Moodie, G. (2006). Vocational education institutions' role in national innovation. research in Post-compulsory Education, 11(2), 131-140.

OECD. (2001). The Well-Being of Nations: The Role of Human and Social Capital. Paris: OECD.

Olazaran, M., Albizu, E., Lavía, C., Otero, B. (2013). Formación profesional, pymes e innovación en Navarra. Cuadernos de Gestión, 13(1), 15-40, D0I: $10.5295 / \mathrm{cdg} .110290 \mathrm{mo}$.

Olazaran, M., Brunet, I. (2013). Entorno regional y Formación Profesional: Los casos de Aragón,
Asturias, Cataluña, Madrid, Navarra y País Vasco. Tarragona: URV Publicacions.

Pérez Díaz, V., Rodríguez, J. (2002). La educación profesional en España. Madrid: Fundación Santillana.

Real Decreto-ley 4/2015. (2015). Real Decreto-ley $4 / 2015$, de 22 de marzo, para la reforma urgente del Sistema de Formación Profesional para el Empleo en el ámbito laboral. Boletín Oficial del Estado 70 (23 de marzo, 25019-25059.

Robertson, P., Patel, P. (2007). New wine in old bottles: technological diffusion in developed economies. Research Policy, 36 , 708-721.

Robertson, P., Smith, K., von Tuzelmann, N. (2009). Innovation in low- and medium-technology industries. Introduction. Research Policy, 38(3), 441-446.

Rosenfeld, S. (1998b). Community college-cluster connections: Specialization and competitiveness in the US and Europe. New York: Community CoIlege Research Centre, Columbia University.

Rosenfeld, S. (1998). Technical colleges, Technology deployment and Regional Development. Modena: OECD.

Sandven, T., Smith, K., Kaloudis, A. (2005). Sturcutral change, growth and innovation: The roles of medium and low tech industries, 1980-2000. En H. Kreinsen, Low-Tech Innovation in the Knowledge Economy. Frankfurt: P. Lane.

Storper, M. (1997). The Regional Economy. New York: Guilford Press.

Vargas Sampedro, T. (2015). La formación continua. En C. Torres Albero, España 2015. Situación social (pp. 584-587). Madrid: Centro de Investigaciones Sociológicas.

Velluzi, N. (2010). Community colleges, clusters and competition: A case from Washington Wine Country. Regional Studies, 44(2), 201-214.

Whittingham, K., Ferrier, F., Trood, C. (2004). Vocational education and training and the commercialisation of Australian research. En S. Dawe, Vocational Education and Training and Innovation: Research Readings (pp. 106-117). Adelaida, Australia: NCVER.

Wolter, S. C., Mühlemann, S. (2015). Apprenticeship training in Spain - a cost effective model for firms? Gütersloh, Germany: Bertelsmann Stiftung. 
Wolter, S. C., Ryan, P. (2011). Apprenticeship. En E. Hanushek, S. Machin, L. Woessmann, Handbook of the Economics of Education, vol. 3 (pp. 521-576). Elsevier.

\section{NOTAS BIOGRÁFICAS}

Beatriz Otero Gutiérrez (Santander, 1978) es doctora en Sociología por la UPV/EHU y profesora adjunta en el departamento de Sociología y Trabajo Social de la misma universidad. Ha participado en diferentes proyectos y publicaciones en el ámbito de los sistemas de I+D, la innovación y la educación, siendo el análisis de datos e indicadores de ciencia y tecnología un área de interés en sus investigaciones.

Cristina Lavía Martínez (Bilbao, 1963) es doctora en Sociología por la Universidad de Deusto y actualmente profesora titular en el Departamento de Sociología y Trabajo Social de la Universidad del País Vasco (UPV/EHU). Sus líneas de investigación y publicación se centran en las metodologías cuantitativas, el desarrollo de nuevos indicadores sociales así como en el análisis de sistemas de innovación.

Mikel Olazaran Rodríguez (Pamplona, 1963) es doctor en Sociología por la Universidad de Edimburgo (1991). Trabaja como profesor titular en el departamento de Sociología y Trabajo Social de la UPV/EHU. Ha realizado investigaciones y publicaciones en torno a la innovación, las organizaciones y la educación. Entre 2005 y 2012 fue decano del Colegio Oficial de Doctores y Licenciados en Ciencias Políticas y Sociología de Navarra.

Eneka Albizu Gallastegi (Bilbao, 1963) es doctor en Economía /UPV/EHU), catedrático en la Facultad de Relaciones Laborales y Trabajo Social y director del Instituto de Economía Aplicada a la Empresa. Ocupa un puesto de catedrático en la Escuela Universitaria de Relaciones Laborales de la misma universidad. Sus áreas de interés investigador pivotan, fundamentalmente, sobre la innovación, tanto en lo concerniente al cambio y aprendizaje organizativo, como a la gestión de personas. 